\title{
Cytokine IL-6 is required in Citrobacter rodentium infection-induced intestinal Th17 responses and promotes IL-22 expression in inflammatory bowel disease
}

\author{
LEI LI ${ }^{1,2^{*}}$, QING-GUO SHI ${ }^{3 *}$, FANG LIN ${ }^{2}$, YU-GUANG LIANG ${ }^{4}$, LI-JIAN SUN ${ }^{2}$, JIN-SONG MU ${ }^{2}$, \\ YONG-GANG WANG ${ }^{2}$, HAI-BIN SU ${ }^{2}$, BIAO XU ${ }^{2}$, CHENG-CHENG JI ${ }^{2}$, \\ HUI-HUANG HUANG ${ }^{2}, \mathrm{KE} \mathrm{LI}^{2}$ and HUI-FEN WANG ${ }^{2}$
}

${ }^{1}$ The Medical College of Chinese PLA and PLA General Hospital, Beijing 100853; ${ }^{2}$ Department of Intensive Care Unit,
The 302 Military Hospital, Beijing $100039 ;{ }^{3}$ Department of Biotechnology, Academy of Military Medical Sciences;
${ }^{4}$ Department of Pharmacology, Affiliated Hospital of Academy of Military Medical Sciences, Beijing 100071, P.R. China

Received May 8, 2013; Accepted November 25, 2013

DOI: $10.3892 / \mathrm{mmr} .2014 .1898$

\begin{abstract}
Citrobacter rodentium (C. rodentium) infection is a widely used murine model to mimic human enteric bacteria infection and inflammatory bowel disease (IBD). In this model, interleukin (IL)-17A plays critical roles in increasing chemokine and cytokine production in various tissues to recruit innate cells, including monocytes and neutrophils, to the local site of infection. However, the source of IL-17A remains unclear, as the majority of cell types produce IL-17A, including intestinal endothelium cells, innate immune cells and $\mathrm{CD}^{+} \mathrm{T}$ cells in disease development. In the current study, wild-type B6 mice were treated with $C$. rodentium and the $\mathrm{CD}^{+} \mathrm{Th} 17$ cell subset was observed as being specifically increased in Peyer's patches (PP), but not in mesenteric draining lymph nodes. Furthermore, the research suggested that the differentiation and activation of Th17 cells in PP were dependent on the inflammatory cytokine IL-6, as blocking IL-6 signaling with neutralizing antibodies decreased Th17 cells and resulted in the mice being more susceptible to $C$. rodentium infection. These results confirmed that the Th17 cell subset was specifically activated in PP and demonstrated that IL-6 is required in Th17 cell activation, which are important to the clinical treatment of IBD.
\end{abstract}

\section{Introduction}

Citrobacter rodentium ( $C$. rodentium) is a predominantly mucosal enteric murine pathogen used in murine models of

Correspondence to: Dr Ke Li or Dr Hui-Fen Wang, Department of Intensive Care Unit, The 302 Military Hospital, Beijing 100039, P.R. China

E-mail: keli2004@gmail.com

E-mail:wanghuifen@medmail.com.cn

${ }^{*}$ Contributed equally

Key words: Citrobacter rodentium, inflammatory bowel disease, Th17 cells, interleukin-6 enteropathogenic Escherichia coli (E. Coli) and enterohemorrhagic $E$. coli infection in humans (1-3). C. rodentium infection in the colon surface results in epithelial hyperplasia and mucosal inflammation (4) and its colonization of the colon reaches its peak 7-10 days post inoculation (DPI) (5), followed by weight loss and diarrhea in association with crypt hyperplasia in mice and returns to normal by 14-25 DPI (6). It is also well established that $C$.rodentium infection induces significant mucosal infiltration of lymphocytes, macrophages, neutrophils and mast cells in the colon (7), goblet cell loss (2) and local and systemic inflammatory cytokines disorders $(8,9)$.

Although innate cells that express Toll-like receptors, including $\mathrm{CD}_{11 \mathrm{~b}^{+}}$myeloid cells, are known to play a major role in host defense against $C$. rodentium infection (9-11), adaptive immune cells are also involved in the eradication of $C$. rodentium infection (12). The roles of B and T cells with regard to protection against $C$. rodentium infection has been previously characterized $(13,14)$. In the mice lacking antibodies production showed a higher susceptibility to $C$. rodentium infection compared with the wild-type mice. It is commonly accepted that $\mathrm{T}$ cells promote innate immune responses mostly by providing pro-inflammatory cytokines, however, in a previous study, $\mathrm{T}$ cells were observed to be involved in B cell dependent $C$. rodentium antigen specific antibody production and interacted with B cells directly (15). Pro-inflammatory cytokines, including interleukin (IL)-17A, IL-21 and IL-17A-induced IL-22 were shown to be crucial for the host defense against extracellular bacteria (16) and these cytokines were primarily secreted by Th17 subsets (17). In addition, it was previously shown that segmented filamentous bacteria (SFB), another microorganism that colonizes in the intestines, was sufficient to induce functional Th17 cells in the lamina propria (18). However in the model of $C$. rodentium infection, it was reported that IL-17A in this model was produced by innate cell subsets, including innate lymphoid tissue inducer cells (LTi cells), which was different from intestinal Th17 cells. Whether $C$. rodentium infection in mice increases $\mathrm{CD}^{+}$Th17 cell subset in the intestinal tissue remains unclear. 
To identify the functions of $C$. rodentium infection in Th17 subset differentiation in the small intestine, $C$. rodentium was inoculated into 5-week-old wild-type B6 mice and the percentage of Th17 cell subsets in various tissues was observed. The results suggested that Th17 subset expression specifically increased in Peyer's patches (PP) but did not alter in mesenteric draining lymph nodes. The mechanistic studies suggested that the inflammatory cytokine IL- 6 was required in Th17 cell differentiation in PP as treatment with anti-IL-6 neutralizing antibodies reduced the Th17 subset percentage and aggravated the clinical manifestation of colitis. Furthermore, these intestinal Th17 cells were functional in innate responses to $C$. rodentium infection by inducing IL-22 production but not in promoting immunoglobulin $\mathrm{A}(\operatorname{Ig} \mathrm{A})$ production in the small intestine.

\section{Materials and methods}

Reagents and mice. C. rodentium strain DBS100 (catalog no. 51459) American Type Culture Collection (Manassas, VA, USA) was cultured in LB medium for $6 \mathrm{~h}$ at $37^{\circ} \mathrm{C}$ with agitation. Following $6 \mathrm{~h}$, the bacterial density was assessed using absorbance at an optical density of $600 \mathrm{~nm}$ and confirmed by the plating of serial dilutions.

Five-week-old mice were orally inoculated with $1 \times 10^{9}$ colony forming units ( $\mathrm{cfu}$ ) of $C$. rodentium using a gavage needle. The body weights and the bacterial concentrations in the feces were assessed for 3 weeks subsequent to inoculation. $\mathrm{PP}$ and small intestine tissues were collected for quantitative polymerase chain reaction (PCR) and FACS analysis.

Bacterial quantification. Fresh fecal pellets were collected from the mice and dissolved in phosphate-buffered saline (PBS) at a concentration of $100 \mathrm{mg} / \mathrm{ml}$. The bacterial colonies were quantified $24 \mathrm{~h}$ following the start of the culture.

Serum samples. Whole blood was collected from mice infected with $C$. rodentium. Serum samples were obtained by centrifugation at $16,000 \mathrm{x} \mathrm{g}$ for $10 \mathrm{~min}$ and stored at $-20^{\circ} \mathrm{C}$ until use in subsequent experiments. Serum total IgA was detected using a mouse total IgA ELISA kit (Tiangen, Beijing, China).

Intracellular cytokine staining. The intracellular expression of IL-17-producing $\mathrm{T}$ cells was analyzed using a Cytofix/Cytoperm kit Plus (with GolgiStop; BD Biosciences, San Jose, CA, USA), according to the manufacturer's instructions. In brief, lymphocytes obtained from spleens, MLNs or PP were incubated with $50 \mathrm{ng} / \mathrm{ml} \mathrm{PMA}, 5 \mu \mathrm{M}$ calcium ionophore A23187 (both from Sigma-Aldrich, St. Louis, MO, USA) and GolgiStop at $37^{\circ} \mathrm{C}$ for $4 \mathrm{~h}$. Surface staining was performed with anti-CD4-PerCP/Cy5.5 (BioLegend, San Diego, CA, USA) for $20 \mathrm{~min}$ at $4^{\circ} \mathrm{C}$, the cells were permeabilized with Cytofix/Cytoperm solution for $20 \mathrm{~min}$ at $4^{\circ} \mathrm{C}$ and intracellular cytokine staining was performed with anti-IL-17A-Alexa Fluor 647 (BD Biosciences).

Flow cytometry. The Abs used for flow cytometry included anti-CD4-PerCP/Cy5.5, anti-TCRb-FITC (BioLegend) and anti-IL-17A-PE (eBioscience, San Diego, CA, USA), according to the manufacturer's instructions. Data were obtained using a FACS LSR II (BD Biosciences) and analyzed using FlowJo software (Tree Star, Inc., Ashland, OR, USA).

Quantitative PCR. Total RNA was extracted with TRIzol reagent (Invitrogen Life Technologies, Carlsbad, CA, USA) according to the manufacturer's instructions. Reverse transcription was performed with a High Capacity cDNA Reverse Transcription kit (Applied Biosystems, Tokyo, Japan) according to the manufacturer's instructions. Quantitative PCR was performed with SYBR-Green PCR Master Mix (Applied Biosystems) according to the manufacturer's instructions. The reaction conditions consisted of 40 cycles of two-stage PCR consisting of denaturation at $95^{\circ} \mathrm{C}$ for $15 \mathrm{sec}$ and annealing at $60^{\circ} \mathrm{C}$ for $1 \mathrm{~min}$ following an initial denaturation step of $95^{\circ} \mathrm{C}$ for $10 \mathrm{~min}$. The primer sequences used were: mouse interferon- $\gamma(\mathrm{IFN}-\gamma)$, forward: 5'-TCAAGTGG CATAGATGTGGAAGAA-3' and reverse: 5'-TGGCTCTGC AGGATTTTCATG-3'; IL-17A, forward: 5'-ATCAGGACGC GCAAACATG-3' and reverse: 5'-TGATCGCTGCTGCC TTCAC-3'; and mouse $\beta$-actin, forward: 5'-AGAGGGAAA TCGTGCGTGAC-3' and reverse: 5'-CAATAGTGATGAC CTGGCCGT-3'. To allow comparisons of mRNA expression levels, the real-time PCR data were analyzed with the $\Delta \Delta \mathrm{Ct}$ method and normalized to the amount of $\beta$-actin cDNA as an endogenous control.

Fecal IgA. Fresh fecal pellets were collected from the mice and dissolved in PBS at a concentration of $100 \mathrm{mg} / \mathrm{ml}$. Fecal $\operatorname{IgA}$ was examined with a mouse IgA-detecting kit (Tiangen).

Statistical analysis. Data are expressed as mean $\pm \mathrm{SE}$ and statistical significance was analyzed using the Student's t-test. $\mathrm{P}<0.05$ was considered to indicate a statistically significant difference.

\section{Results}

$C$. rodentium infection induces inflammatory bowel disease (IBD) in mice. C. rodentium infection induced IBD in mice is a commonly used animal model to study innate cell responses and inflammatory signaling in intestinal inflammation in vivo. To examine the importance of $\mathrm{T}$ cells in IBD caused by $C$. rodentium, wild-type female $\mathrm{B} 6$ mice of various ages were infected with $C$. rodentium at $4 \times 10^{9} \mathrm{cfu} /$ mouse. A survival curve, body weight loss and clinical manifestation of colitis were monitored during a course of 3 weeks following infection and young mice ( 3 weeks) were observed to be highly susceptible to $C$. rodentium infection as they cannot survive colitis infection (Fig. 1). C. rodentium was capable of inducing IBD in the mice between 5 and 8 weeks old, however, the older mice ( 8 weeks) exhibited less susceptibility compared with the 5-week-old mice (Fig. 2), as they lost significantly less body weight (Fig. 3).

Considering that innate immune cells were immature in newborn mice and the newborn mice did not have sufficient amounts of $\operatorname{Ig} \mathrm{A}$ antibody secretion on the surface of the intestine, which was significant in the host against microbe infection (14), female mice of 5 weeks age were selected for the present study. The body weight continued to decrease in the first 10 days following $C$. rodentium infection and visible 


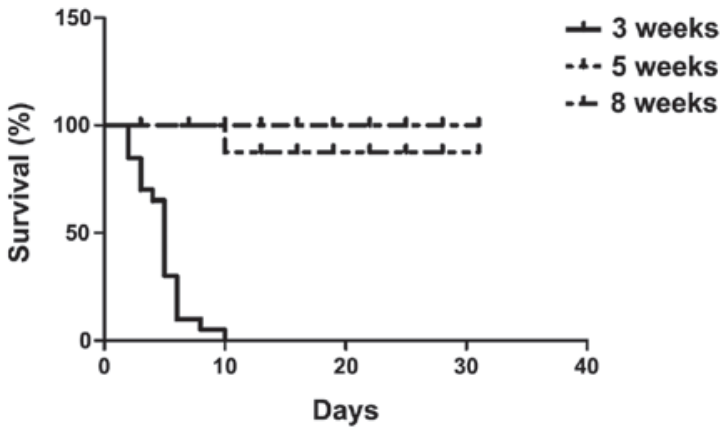

Figure 1. Survival curve of different age female mice infected with C. rodentium. Mice were orally infected with $\mathrm{C}$. rodentium at $4 \times 10^{9} \mathrm{cfu} /$ mouse. Percentage of survival was monitored during a course of 3 weeks following infection $(\mathrm{n}=20)$. Cfu, colony forming units; C. rodentium, Citrobacter rodentium

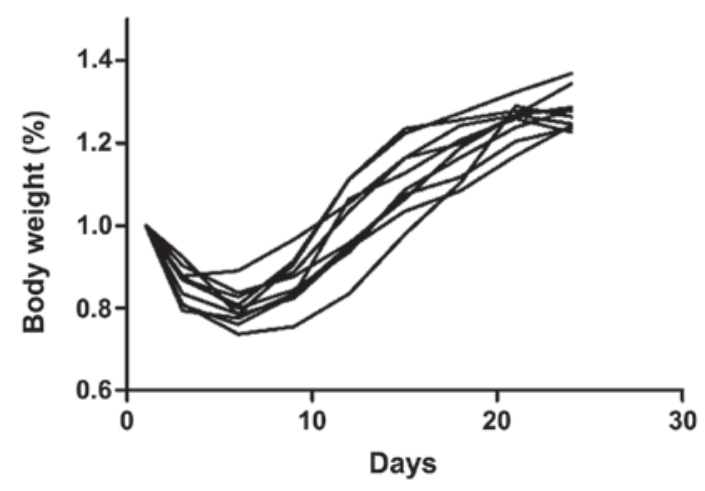

Figure 2. Body weight loss of 5-week-old female mice following C. rodentium infection $(\mathrm{n}=10)$. C. rodentium, Citrobacter rodentium.

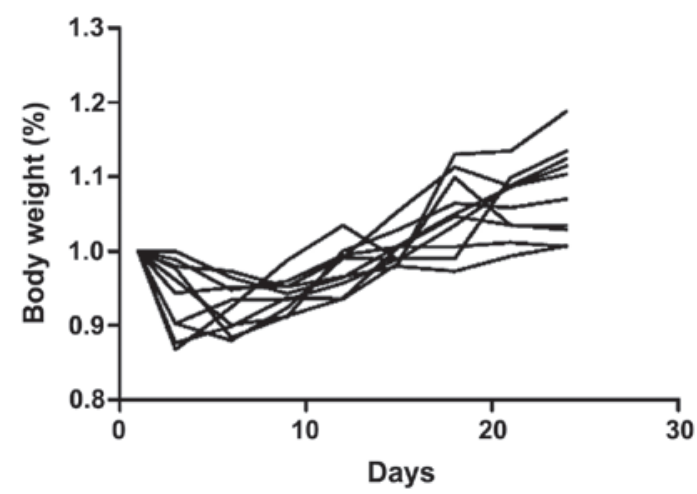

Figure 3. Body weight loss of 8-week-old female mice following C. rodentium infection $(\mathrm{n}=10)$. C. rodentium, Citrobacter rodentium.

colonic inflammation, including diarrhea and loss of appetite was observed. Loss of appetite was observed in the first 1 week following $C$. rodentium infection. Bacterial counts in fecal pellets showed that the amount of $C$. rodentium reached the peak at day 7 and returned to normal 2 weeks following infection (Fig. 4). However, there was no significant difference observed between the male and female groups (data not shown).

C. rodentium infection increased the Th17 subset specifically in PP. The pro-inflammatory cytokine IL-17A is critical

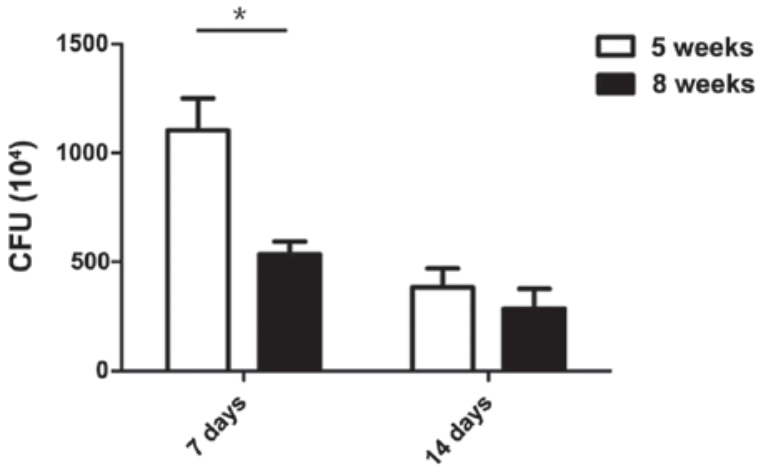

Figure 4. Cfu of C. rodentium in fecal pellets of infected mice. Cfu, colony forming units; $C$. rodentium, Citrobacter rodentium.
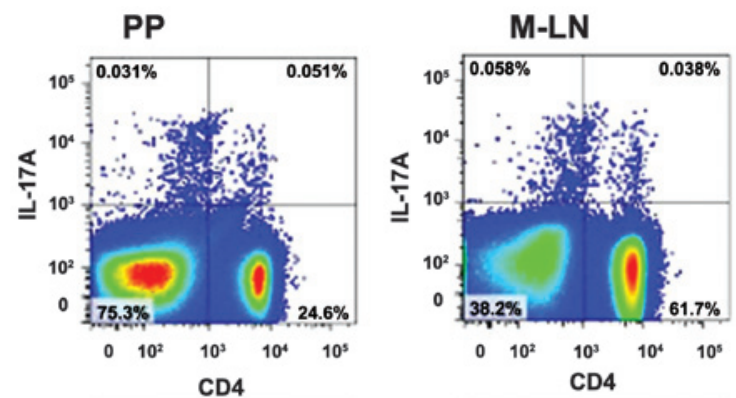

CTRL
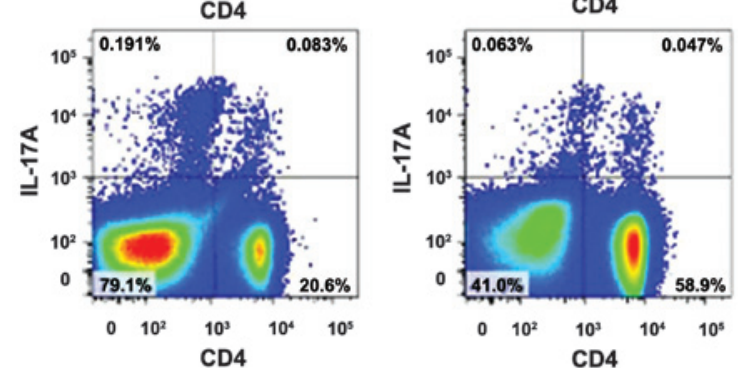

Infected

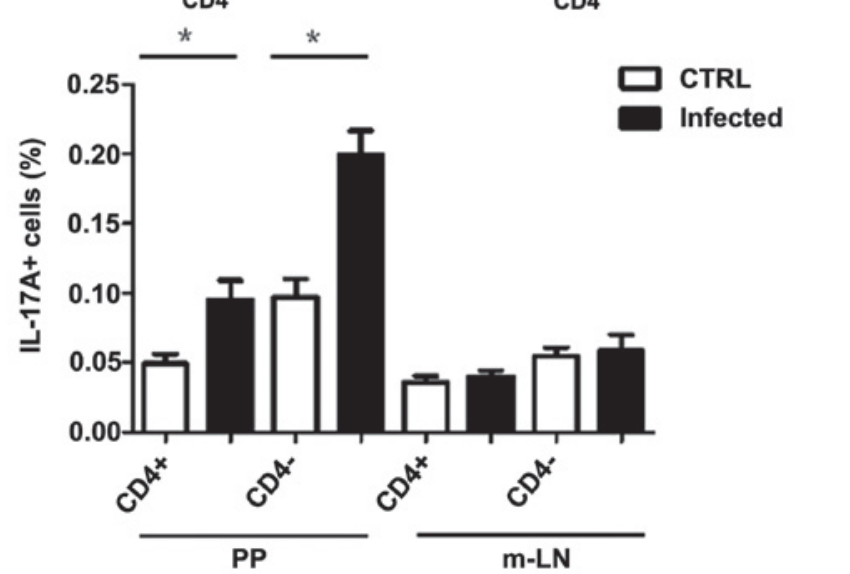

Figure 5. Interleukin (IL)-17A-producing cells in Peyer's patches and m-LNs $(\mathrm{n}=6)$.

for host response to the invasion of the immune system by extracellular pathogens $(19,21)$ by increasing chemokine production in various tissues to recruit innate cells, including monocytes and neutrophils to the site of local infection. In the model of $C$. rodentium infection, it was reported that C. rodentium-induced colitis triggers strong IL-17A secretion in the small intestine in mice (17). Recent studies suggested that IL-17A was primarily secreted by LTi cells and 'innate' 
CTRL

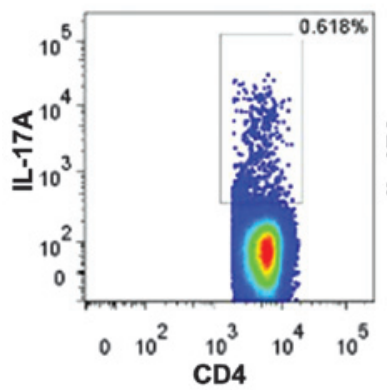

Infected+anti-IL-6

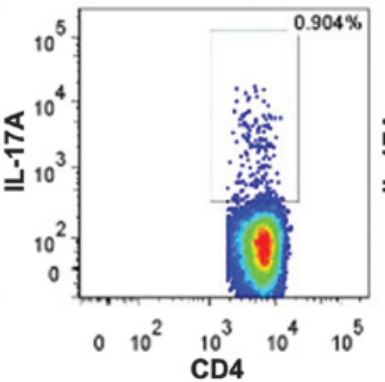

Infected

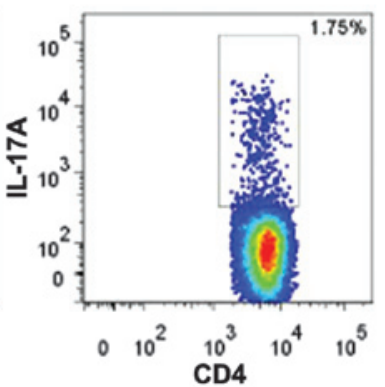

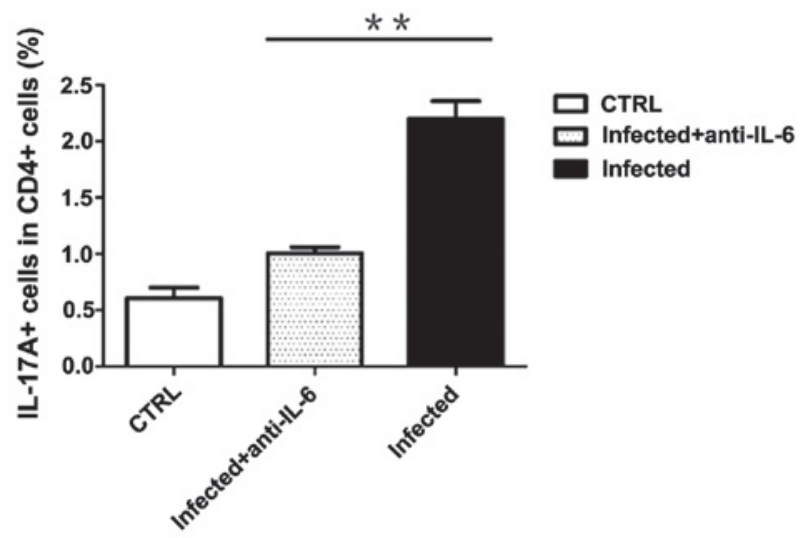

Figure 6. Anti-IL-6 antibodies treatment reduces IL-17A-producing Th17 cell subset in Peyer's patches of $C$. rodentium-infected mice ( $\mathrm{n}=6$ ). IL, interleukin; C. rodentium, Citrobacter rodentium.

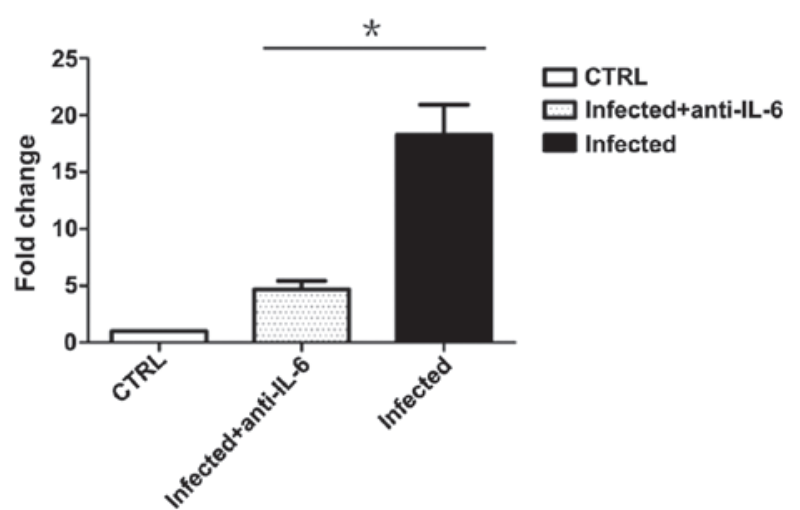

Figure 7. IL-22 expression levels in intestinal tissues (n=6). IL, interleukin.

Th17 cells $(16,20)$. However, whether $C$. rodentium infection was capable of increasing the classic Th17 subset in vivo remains unclear.

To confirm intestinal Th17 differentiation in colitis, 5-week-old female B6 wild-type mice were infected with C. rodentium $\left(4 \times 10^{9} \mathrm{cfu} /\right.$ mouse) or inactivated $C$. rodentium as the control group. Mice were sacrificed 7 days following infection and Th17 cells group were examined using intercellular staining of the cytokine IL-17A. IL-17A producing $\mathrm{CD}^{+} \mathrm{T}$ cells group were observed to increase in PP, but no significant change in mesenteric draining lymph nodes was observed. Notably, the IL-17-producing non-T cell-group (TCRb-CD4 ${ }^{-}$) also increased in PP and did not change in the m-LN group (Fig. 5) and these innate cells produced

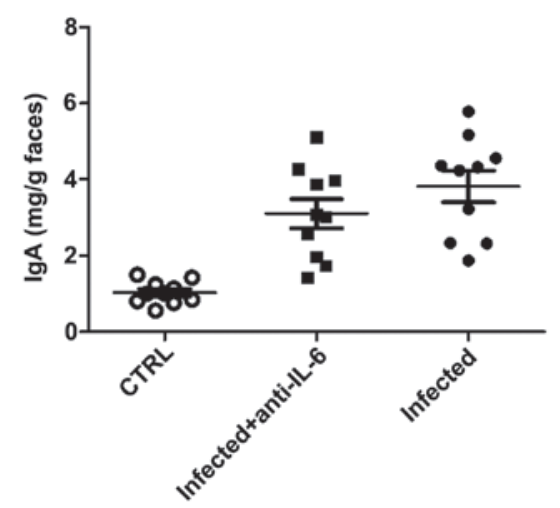

Figure 8. IgA levels in feces of C. rodentium infected mice $(\mathrm{n}=10) . \operatorname{Ig} \mathrm{A}$, immunoglobulin A; C. rodentium, Citrobacter rodentium.

increased IL-17A compared with CD4+ Th17 cells, which was consistent with a previous report (16). However, the innate cells cannot protect the host from $C$. rodentium infection, as it was observed that these mice lack $\mathrm{T}$ cells and with functional innate cells, including Rag1 deficient mice, exhibited a higher susceptibility to IBD compared with the wild-type mice. In addition, the mice with colitis also exhibited increased PP and shorter length of the small intestine when compared with the control group (data not shown).

IL-6 is required in intestinal Th17 differentiation in C. rodentium-induced colitis model. The IL-6 cytokine was required in Th17 differentiation and activation in vitro and in vivo. Early induction of IL-6 secretion was critical in the host response 
to enteric microbial infection. $C$. rodentium-infected female B6 wild-type mice were treated with anti-IL-6 neutralizing antibodies or control IgG to block IL-6 signaling 0 and 3 days following infection. These mice were sacrificed 7 days following infection and cells from PP were collected for intercellular cytokine analysis. The results suggest that treatment with anti-IL-6 neutralizing antibodies was capable of decreasing IL-17A-producing $\mathrm{CD}^{+}{ }^{+} \mathrm{T}$ cells in PP (Fig. 6). However, the percentage of Th17 cells in PP remained higher than that in mice treated with inactivated $C$. rodentium. Similarly, anti-IL-6 neutralizing antibody-treated mice exhibited more severe clinical symptoms compared with the control IgG-treated group. However, results of the statistical analysis of clinical scores and length of the small intestine revealed no significant difference (data not shown).

IL-17A production in non-T cells was investigated in this model and no significant difference was observed between anti-IL-6 neutralizing treatment and the control group, which suggesting that IL- 6 was not required in the activation of IL-17A-producing innate cells.

Intestinal Th17 cells promotes IL-22 and IgA production in the host defense against $C$. rodentium infection. IL-22 is a multi-functional cytokine with diverse biological activities, including tissue repair and pathogen defense in the model of IBD in mice. In addition, it was previously reported that $\operatorname{IgA}$ production by B cells was dependent on T-helper cells (15). To determine whether these increasing intestinal Th17 cells were involved in colitis, mRNA expression levels of IL-22 were examined in the small intestine tissue and $\operatorname{IgA}$ in the feces in anti-IL-6 neutralizing antibodies or IgG control-treated mice 7 days following $C$. rodentium infection. Results suggest that anti-IL-6 neutralizing antibody-treated mice exhibited a lower level of IL-22 expression in the small intestine (Fig. 7), however, the IL-22 protein was not detected in the small intestine tissue lysis and intestinal lavage fluid (data not shown). There was no significant difference in IgA levels in feces between anti-IL-6 neutralizing antibody-treated mice and the control group (Fig. 8), suggesting that intestinal Th17 cells was involved in the defense against $C$. rodentium infection by promoting IL-22 expression in the small intestine.

\section{Discussion}

A Th17 cell subset was identified in multiple auto-immune disease models in mice and humans, however, the differentiation and activation of these cells remains unclear. It was reported that the Th17 subset in intestinal lamina propria was dependent on commensal microbes with specialized properties since, in germ-free (GC) mice, Th17 cells failed to develop $(15,22)$. In addition, in the study of SFB mono-colonized mice, SFB was shown to sufficiently direct the accumulation of Th17 cells in the intestinal lamina propria by unknown mechanisms.

$C$. rodentium is a predominantly mucosal enteric murine pathogen that is widely used in murine models of intestinal colitis. These models are primarily mediated by pro-inflammatory cytokine IL-17A. It is commonly accepted that LTi-associated innate cells and $\gamma \Delta \mathrm{T}$ cells are the major sources in C. rodentium-infected Rag1-deficient mice $(23,24)$, however, Th17 cells and their roles in $C$. rodentium-infected wild-type mice may be different from Rag1-deficient mice. Elucidating the sources of IL-17A and the differentiation and activation of Th17 cells in intestine is essential for understanding the etiology of IBD.

In the current study, IL-17A-producing Th17 cell subsets in $C$. rodentium infection-induced colitis model were investigated and the Th17 cell subset was observed to increase specifically in PP. Although IL-17A are produced by a number of types of cells, including intestinal endothelium cells and CD4 ${ }^{+}$ lymphoid tissue inducer cells, Th17 cells were hypothesized to play a major role in IBD as decreasing Th17 percentage in PP led to high susceptibility to $C$. rodentium infection in mice. Notably, the innate cells secreted increased IL-17A compared with Th17 cells in this model, but were unable to protect the host from the disease.

The inflammatory cytokine IL-6 is required in Th17 cell differentiation and activation in vitro and a large amount of IL-6 secretion may be detected in intestinal tissues. Therefore, IL-6 was hypothesized to be required in $C$. rodentium infection-induced colitis model. In the current model, treatment with anti-IL-6 neutralizing antibodies in $C$. rodentium-infected mice were efficient to decrease the Th17 subset in PP and aggravate the symptoms of the disease. Furthermore, the Th17 cells were considered to have a physical function in inducing IL-22 secretion in intestinal tissue according to the quantitative PCR data, however, IL-22 protein was not observed in the intestinal surface and feces. The results also suggested that Th17 cells had no effect on IgA production by B cells, although it was recently reported that Th17 cells in PP was responsible for the induction of T cell-dependent IgA (25).

\section{Acknowledgements}

This study was supported by a Youth Innovation Fund of the First Affiliated Hospital of Zhengzhou University and grants from the National Grand Program on Key Infectious Disease (no. 2008ZX1002-005-6) and the National Natural Science Foundation of China (no. 81141106).

\section{References}

1. Mundy R, MacDonald TT, Dougan G, Frankel G and Wiles S: Citrobacter rodentium of mice and man. Cell Microbiol 7: 1697-1706, 2005.

2. Luperchio SA and Schauer DB: Molecular pathogenesis of Citrobacter rodentium and transmissible murine colonic hyperplasia. Microbes Infect 3: 333-340, 2001.

3. Borenshtein D, McBee ME and Schauer DB: Utility of the Citrobacter rodentium infection model in laboratory mice. Curr Opin Gastroenterol 24: 32-37, 2008.

4. Kim YG, Kamada N, Shaw MH, Warner N, Chen GY, Franchi L and Núñez G: The Nod2 sensor promotes intestinal pathogen eradication via the chemokine CCL2-dependent recruitment of inflammatory monocytes. Immunity 34: 769-780, 2011.

5. Johnson E and Barthold SW: The ultrastructure of transmissible murine colonic hyperplasia. Am J Pathol 97: 291-313, 1979.

6. Morgan ET, Goralski KB, Piquette-Miller M, et al: Regulation of drug-metabolizing enzymes and transporters in infection, inflammation, and cancer. Drug Metab Dispos 36: 205-216, 2008.

7. Neurath MF, Weigmann B, Finotto S, et al: The transcription factor T-bet regulates mucosal $\mathrm{T}$ cell activation in experimental colitis and Crohn's disease. J Exp Med 195: 1129-1143, 2002.

8. Nenci A, Becker C, Wullaert A, et al: Epithelial NEMO links innate immunity to chronic intestinal inflammation. Nature 446: 557-561, 2007. 
9. Gibson DL, Ma C, Bergstrom KS, Huang JT, Man C and Vallance BA: MyD88 signalling plays a critical role in host defence by controlling pathogen burden and promoting epithelial cell homeostasis during Citrobacter rodentium-induced colitis. Cell Microbiol 10: 618-631, 2008.

10. Gibson DL, Ma C, Rosenberger CM, et al: Toll-like receptor 2 plays a critical role in maintaining mucosal integrity during Citrobacter rodentium-induced colitis. Cell Microbiol 10: 388-403, 2008.

11. Lebeis SL, Bommarius B, Parkos CA, Sherman MA and Kalman D: TLR signaling mediated by MyD88 is required for a protective innate immune response by neutrophils to Citrobacter rodentium. J Immunol 179: 566-577, 2007.

12. Eckmann L: Animal models of inflammatory bowel disease: lessons from enteric infections. Ann N Y Acad Sci 1072: 28-38, 2006.

13. Vallance BA, Deng W, Knodler LA and Finlay BB: Mice lacking $\mathrm{T}$ and $\mathrm{B}$ lymphocytes develop transient colitis and crypt hyperplasia yet suffer impaired bacterial clearance during Citrobacter rodentium infection. Infect Immun 70: 2070-2081, 2002.

14. Shiomi H, Masuda A, Nishiumi S, et al: Gamma interferon produced by antigen-specific $\mathrm{CD}^{+} \mathrm{T}$ cells regulates the mucosal immune responses to Citrobacter rodentium infection. Infect Immun 78: 2653-2666, 2010.

15. Bry L and Brenner MB: Critical role of T cell-dependent serum antibody, but not the gut-associated lymphoid tissue, for surviving acute mucosal infection with Citrobacter rodentium, an attaching and effacing pathogen. J Immunol 172: 433-441, 2004.

16. Sonnenberg GF, Monticelli LA, Elloso MM, Fouser LA and Artis D: CD4(+) lymphoid tissue-inducer cells promote innate immunity in the gut. Immunity 34: 122-134, 2011.
17. Ishigame $\mathrm{H}$, Kakuta $\mathrm{S}$, Nagai $\mathrm{T}$, et al: Differential roles of interleukin-17A and -17F in host defense against mucoepithelial bacterial infection and allergic responses. Immunity 30: 108-119, 2009.

18. Ivanov II, Atarashi K, Manel N, et al: Induction of intestinal Th17 cells by segmented filamentous bacteria. Cell 139: 485-498, 2009.

19. Chiricozzi A, Guttman-Yassky E, Suarez-Fariñas M, et al: Integrative responses to IL-17 and TNF- $\alpha$ in human keratinocytes account for key inflammatory pathogenic circuits in psoriasis. J Invest Dermatol 131: 677-687, 2011

20. Geddes K, Rubino SJ, Magalhaes JG, et al: Identification of an innate $\mathrm{T}$ helper type 17 response to intestinal bacterial pathogens. Nat Med 17: 837-844, 2011

21. Miossec P, Korn T and Kuchroo VK: Interleukin-17 and type 17 helper T cells. N Engl J Med 361: 888-898, 2009.

22. O'Dell JR, Elliott JR, Mallek JA, et al: Treatment of early seropositive rheumatoid arthritis: doxycycline plus methotrexate versus methotrexate alone. Arthritis Rheum 54: 621-627, 2006.

23. Sartor RB: Microbial influences in inflammatory bowel diseases. Gastroenterology 134: 577-594, 2008.

24. Buonocore S, Ahern PP, Uhlig HH, Ivanov II, Littman DR, Maloy KJ and Powrie F: Innate lymphoid cells drive interleukin-23-dependent innate intestinal pathology. Nature 464: 1371-1375, 2010

25. Hirota K, Turner JE, Villa M, Duarte JH, Demengeot J, Steinmetz OM and Stockinger B: Plasticity of TH17 cells in Peyer's patches is responsible for the induction of T cell-dependent IgA responses. Nat Immunol 14: 372-379, 2013. 